\title{
I paratesti dell'Electra di Lazare de Baïf (1537)
}

La riflessione di un umanista sul problema del volgarizzamento dei testi teatrali classici

\section{Filippo Fassina}

\section{(2) OpenEdition}

\section{Journals}

Edizione digitale

URL: https://journals.openedition.org/eve/719

DOI: $10.4000 /$ eve. 719

ISSN: 2425-1593

\section{Editore:}

Université de Savoie, Université Jean Moulin - Lyon 3

\section{Notizia bibliografica digitale}

Filippo Fassina, «l paratesti dell'Electra di Lazare de Baiff (1537)», Corpus Eve [Online], Area francese, online dal 18 octobre 2013, consultato il 16 avril 2022. URL: http://journals.openedition.org/eve/719 DOI: https://doi.org/10.4000/eve.719

Questo documento è stato generato automaticamente il 16 avril 2022

(c) Tous droits réservés 


\section{I paratesti dell'Electra di Lazare de Baïf (1537)}

La riflessione di un umanista sul problema del volgarizzamento dei testi teatrali classici

Filippo Fassina

\section{NOTIZIA}

Lazare de Baïf, Tragedie de Sophoclés intitulee Electra, ms. Soissons, Venezia, Biblioteca Marciana, XXIV [=235] (CIV. 8: fondo Recanati IX), paratexte, fo. 1r-2r, 44v ; Paris, Estienne Roffet, 1537, p. 1r-3r.

\section{Presentazione dell'autore}

1 Lazare $\mathrm{I}^{\mathrm{er}}$ de Baïf ${ }^{1}$ nacque alla Cour des Pins, una proprietà della famiglia Mangé, situata tra La Flèche e Bazouges, passata alla famiglia de Baïf in seguito al matrimonio di Ysabeau de Mangé con Antoine de Baif, avvenuto prima del 1434. I biografi sono incerti sulla data di nascita di Lazare: il 1490, indicato in epoca moderna, sembra essere un errore. Infatti, nella dedica al cardinale Jean de Lorraine del De re vestiaria - opera del 1526 - Lazare si dichiara appena trentenne: questa testimonianza posticipa la data di nascita quasi sicuramente al 1496.

2 Ricevette gli ordini minori, che gli permettevano di accedere a benefici ecclesiastici, e studiò diritto. Fu avvocato al Parlamento, ma, dopo aver conosciuto Christophe de Longueil, anch'egli studioso di diritto, decise insieme all'amico di abbandonare tali studi per apprendere la lingua greca. I due si recarono a Roma per divenire allievi di Giovanni Lascaris e nel 1516 entrarono nel Collegio del Quirinale rimanendovi, con buona probabilità, fino al 1519. Tornato in Francia, Lazare si dedicò all'insegnamento del diritto e delle lettere presso l'università di Angers, poi si ritirò a Les Pins. Fu 
durante questo periodo che iniziò la stesura di due dei suoi trattati latini, il De re vestiaria e il De re navali.

3 Nel 1525, Lazare era al seguito del cardinale Jean de Lorraine che si apprestava a portare aiuto al fratello Antoine per sedare una sommossa popolare di matrice luterana (la cosiddetta guerra dei Rustauds). Il rapporto privilegiato di Jean de Lorraine con Francesco I permise a Lazare di ottenere, dopo un breve ritorno a Les Pins, l'incarico di Protonotario (1527). Il 25 giugno del 1529 partì per Venezia per ricoprire l'incarico di ambasciatore di Francia, sempre per conto del sovrano Francesco I. Nel 1531, intanto, fu riedito il De re vestiaria, vide la luce il De vasculorum materiis ac varietate e fu completata la traduzione delle prime quattro Vite di Plutarco (Teseo e Romolo, Licurgo e Numa, di cui solo le prime due sono oggi conosciute ${ }^{2}$ ). In una lettera del 14 novembre 1531 al cardinale Jean de Lorraine, Lazare faceva cenno a un trattatello, fino a oggi non ritrovato, De architectura ${ }^{3}$.

4 Sappiamo che Baïf ebbe un figlio a Venezia, ma poco o nulla si sa della madre. Al figlio fu dato il nome di Jean-Antoine e, per quanto riguarda la data di nascita, situabile nel 1532, sarà lo stesso Jean-Antoine a informarci di avere quarant'anni nel febbraio 1572. Nel 1534 padre e figlio lasciarono Venezia per tornare in Francia. Il 27 marzo $1534 \mathrm{gli}$ fu confermato l'incarico (già affidatogli il 17 novembre 1530, durante il suo servizio in Italia) di conseiller clerc al Parlamento.

Nel 1536, vennero pubblicati in un solo volume, per i tipi di Robert Estienne, i suoi tre trattati latini (il De re vestiaria, il De vasculorum materiis ac varietate e il De re navali) insieme al trattato De coloribus di Antonio Telesio. Nello stesso anno, lavorò con lo stesso editore, Robert Estienne, alla stesura del Thesaurus linguce latince e nel 1537 vide la luce la traduzione in francese dell'Elettra di Sofocle ${ }^{4}$. Lazare collaborò, inoltre, con Denis Lambin per l'edizione di Cicerone e con Budé stesso per la riedizione del De Asse. A partire dal 1535, Charles Estienne compose dei veri e propri riassunti dei trattati di Baif, fatto che ne dimostra la grandissima diffusione.

6 Nel 1538, Lazare iniziò a esercitare l'incarico di maître des requêtes dell'Hôtel du roi. Nel 1540, Baîf fu inviato in Germania alla dieta di Haguenau (e non a quella di Spira, come indicava la maggior parte dei biografi), un'assemblea intesa a conciliare cattolici e protestanti; in realtà, il vero scopo di questa missione era di favorire un'allenaza tra Francesco I e i principi protestanti, in modo da indebolire Carlo $V^{5}$. La missione, tuttavia, fu un insuccesso e Lazare fu sollevato dall'incarico. Il rapporto con Francesco I, comunque, non si incrinò e non solo Lazare fu riconfermato maître des requêtes, ma, nel $1541 \mathrm{fu}$ incaricato di dirimere la controversia esplosa tra la sorella del re, Marguerite de Navarre ed Erard de Grossoles, vescovo di Condom (da qui il nome di 'affaire Condom'), che la accusava di appoggiare l'eresia dei sacramentaires. Inoltre, tra il 1542 e il 1544 tenne alcuni incarichi nella Champagne, in Languedoc e in Poitou. Nel 1544, in seguito alla morte del cugino, Loys de Ronsart, Lazare si occupò dell'educazione del figlio di questi, Pierre Ronsard ${ }^{6}$. Lazare morì nel 1547.

\section{La questione della traduzione}

7 Prima della versione di Lazare de Baif, circolano in Francia alcune versioni latine di tragedie greche a cura di Erasmo (1504-1506) 7 e di Joachim Camerarius ${ }^{8}$. Le traduzioni latine hanno la funzione, dichiarata, di offrire un aiuto ai lettori del testo greco. Erasmo, ad esempio, dichiara di apprestare una traduzione intesa a «versum versui, 
verbum pene verbo reddere» per facilitare la comprensione dell'oscurità di testi difficilissimi, quali, appunto, le tragedie greche ${ }^{9}$. Anche Camerarius si limita a offrire uno strumento di comprensione del testo originario: il che è reso evidente dal fatto che le sue versioni latine si accompagnano col testo greco, posto a fronte, e con un commento.

Questi traduttori di tragedie sono concordi nel denunciare l'obscuritas del testo drammatico antico, delle parti corali in particolare: Erasmo, ad esempio, sottolinea che i cori delle tragedie greche sono talmente obscuri da necessitare di un interprete agguerrito di enigmi quale Edipo, se non addirittura di un interprete di oracoli quale Apollo $^{10}$. Questa difficoltà di comprensione induce a fornire strumenti che facilitino la lettura, ed è anche uno stimolo per il traduttore che ha consapevolezza di cimentarsi in una impresa d'alto profilo letterario e particolarmente meritevole sul piano del linguaggio. Senza contare che, come è stato di recente messo in evidenza ${ }^{11}$, nell'interpretatio della tragedia classica appaiono, più che nelle traduzioni o rielaborazioni di altri generi, gli interventi di reductio del testo antico alla modernità.

In quest'ottica di offrire un ausilio alla lettura dell'originale, si pone Lazare de Baïf, come suggerisce la sottotitolazione della Tragedie: «La dicte Tragedie traduicte du grec dudit Sophoclés en rythme Francoyse, ligne pour ligne, et vers pour vers: en faveur et commodité des amateurs de l'une et l'autre langue». Infatti, come si legge anche nell'affermazione con cui si apre il Prologue: «Sire, pour vous donner aulcunement la congnoissance des inventions des Grecz», Lazare non intende offrire un testo autonomo, che rivaleggi con quello degli antichi, ma soltanto fare intravvedere qualcosa della grandezza poetica dell'originale (il valore fortemente limitativo dell'avverbio aulcunement è, per certo, significativo). D'altronde, la dichiarazione di modestia pronunciata da Lazare - che si autodefinisce simple truchement fidele di Sofocle e, per di più, non suffisamment exercité en l'un et l'aultre langaige - conferma che l'autore si riferisce al suo volgarizzamento da una prospettiva decisamente minimalista.

Tuttavia, se ben si considera il discorso del Prologue, vediamo presente in esso, seppure in nuce, una riflessione di fondo sull'essenza della traduzione - e, in particolare, sul rapporto di quest'ultima con l'originale - che preannuncia ulteriori e più complesse valutazioni. Si tratta, anzitutto, dell'introduzione di una metafora tessile (giocata sull'identificazione traduttore/tessitore), che avrà fortuna nel decennio seguente e che ritroveremo nella teorizzazione di Sébillet, il quale farà ricorso al lessico dell'ourdir (carder, filer, ourdissance, étoffe, couverture, doubleure) per indicare la composizione letteraria e il lavoro del traduttore. Infatti, nella lettera di dedica alla sua versione dell'Ifigenia euripidea, Sébillet descrive, con immagini riferite alla tessitura, l'impresa del tradurre ${ }^{12}$.

11 L'uso del linguaggio metaforico, sia in Lazare che in Sébillet, ha lo scopo di evidenziare la difficoltà di un'impresa che concerne la costruzione linguistica intesa come orditura di tasselli (i lessemi), combinati secondo meccanismi di composizione e scomposizione fondatori di un'icona retorica. Nello stesso tempo, l'immagine della tapisserie in Lazare o della couverture o doubleure in Sébillet riportano al concetto di opera d'arte, che richiede artifizio complesso di esecuzione. Il che è sicuramente un modo di attribuire dignità all'opera di traduzione.

12 Tuttavia, la dichiarazione di inadeguatezza che si accompagna alla metafora della tapisserie in Lazare (con l'affermazione che la translation offre soltanto l'envers dello 
straordinario arazzo tessuto da Sofocle) sottolinea l'inevitabile inferiorità di qualsivoglia traduzione rispetto all'originale tradotto.

Lazare, infine, sempre nel Prologue, accenna a un problema che sarà centrale nei traduttori di tragedie greche (e latine), quello metrico, o meglio della polimetria degli originali. Pur dichiarando la composizione in rima estranea alla sua produzione, egli ritiene essenziale per quanto concerne la versione dell'Elettra, l'attenzione alla versificazione.

Proprio perché vuole essere intermediario fedele (truchement fidele), Lazare denuncia la volontà di rispecchiare la metrica dell'originale greco («j'ay observé les nombres de ses mettres [...] et $\mathrm{y}$ ay adjousté rythme telle quelle»). Evidentemente, questa volontà di ricostruire una polimetria telle quelle è irrealizzabile, se non altro per la perdita, nei moderni, del senso della quantità vocalica.

Tuttavia, la varietà di metri ${ }^{13}$ appare un tentativo di adeguamento all'originale, adeguamento che rappresenta ancora il vanto di Sébillet, il quale, volendo «aviser de [sa] conception» i lettori della sua Iphigéne d'Euripide, insisterà non solo sul fatto di essersi conformato, nel suo modo di scrivere, alla gravité dell'originale, ma soprattutto sul fatto di avere seguito Euripide "quasi à pié levé» nella metrica, rendendo il tetrametro trocaico con l'alessandrino, il trimetro giambico e alcuni versi anapestici con il decasillabo (il verso héroïque) ${ }^{14}$.

\section{Testi : paratesti della Tragedie de Sophoclés intitulee Electra di Lazare de Baïf (1537)}

\section{Criteri di edizione}

Fonte

Dell'Electra di Lazare de Baïf esiste un testo manoscritto e un testo a stampa:

- ms. Soissons, Venezia, Biblioteca Marciana, XXIV [=235] (CIV. 8: fondo Recanati IX); i paratesti si trovano nei ff. $1 \mathrm{r}-2 \mathrm{r}$ e $44 \mathrm{v}$;

- ed. Parigi, Bibliothèque Nationale [Rés. Yb 1057] (paratesti: pp. 1r-3r).

Dall'esame delle varianti è evidente che siamo in presenza di due redazioni diverse e che l'edizione a stampa offre una seconda redazione riveduta e corretta. Per tale motivo, l'edizione critica prende come base il testo della stampa parigina. Quanto ai paratesti, che qui riportiamo, occorre segnalare la seguente collocazione:

- [Titolo]: presente in entrambe le versioni;

- Privilège: presente in entrambe le versioni;

- Diffinition de tragedie: presente in entrambe le versioni (nella stampa in apertura di testo, nel manoscritto in conclusione);

- Prologue: compare solo nel manoscritto;

- Argument: presente in entrambe le versioni;

- Au lecteur: compare solo nella stampa.

Per l'esatta corrispondenza dei testi del ms. e dell'ed. e per la paginazione e per tutte le considerazioni filologiche e la repertoriazione delle varianti: Lazare de Baif, Tragedie de Sophoclés intitulee Electra, a cura di F. Fassina, Vercelli, Mercurio, 2012. 


\section{Criteri di trascrizione} mort d'Agamemnon roy de Mycenes la grand, faicte par sa femme Clytemnestra, et son adultere Egistus. Ladicte Tragedie traduicte du grec dudit Sophoclés en rythme Françoyse, ligne pour ligne, et vers pour vers: en faveur et commodité des amateurs de l'une et l'autre langue. Imprimee à Paris pour Estienne Roffet demourant sur le pont Sainct Michel à l'enseigne de la Rose. 1537. Avec Privilege.

\section{Privilegio}

Il est permis à Estienne Roffet de faire imprimer la Tragedie de Sophoclés soubz le nom d'Electra, traduicte en vulgaire françoys. Et deffences à tous aultres de ne l'imprimer ne exposer en vente dedans deux ans prochainement venans, sur poyne de confiscation de dix livres, et amende arbitraire.

I. I. De mesmes.

\section{Definizione di tragedia}

DIFFINITION DE TRAGEDIE ${ }^{16}$ 
Tragedie est une moralité composee des grandes calamitez, meurtres et adversitez survenues aux nobles et excellentz personnaiges, comme Aias, qui se occist pour avoir esté frustré des armes d'Achillés, Edipus qui se creva les yeulx aprés qu'il luy fut declairé comme il avoit eu des enfans de sa propre mere, aprés avoir tué son pere. Et plusieurs aultres semblables. Tant que Sophoclés en a escript six vingtz: entre lesquelles est ceste presente, intitulee Electra, pour ce qu'elle y est introduicte, et y parle tant bien et virilement, que ung chascun s'en peult donner merveille. Euripidés aussi et plusieurs aultres ont composé pareilles Tragedies. Et la grace d'icelles a anciennement si bien regné, que les roys et princes se mesloyent d'en composer, mesmement Dionysius Roy de Sicile, et Herodés Roy des Perses, et assez d'aultres.

\section{Prologo}

PROLOGUE ${ }^{17}$

Sire, pour vous donner aulcunement la congnoissance des inventions des Grecz et de leur grande et inestimable prudence à bien coucher icelles et mectre par escript, j'ay entrepris de vous translater une tragedie de Sophoclés, laquelle est intitulee Electra. Pource qu'elle est en ce introduicte, et y parle tant bien et virilement que ung chascun s'en peult donner merveille. Mais, Sire, en lisant ceste presente tragedie en françoys ayez s'il vous plaist souvenance de ce que Themistoclés dist au roy des Perses, lequel vouloit parler à luy des affaires de la guerre par truchement et interprete. Auquel fist response luy monstrant l'envers d'une tapisserie que telle estoit l'interpretation d'un langaige comme l'envers d'un tapiz. Par quoy luy supplia qu'il luy pleust donner ung an de terme pour apprendre la langue Persienne. Semblablement vous, Sire, ayez estime que par la mienne translation je n'ay povoir de vous monstrer aultre chose que l'envers de la triumphante et excellente tapisserie de Sophoclés, laquelle en son endroit est tant bien ornee de fleurs d'antique et aultres figures et couleurs que je ne puys penser que depuys mille ans ait esté homme en ce monde qui en peust faire une semblable, telle est l'art de laquelle il a usé pour esmouvoir les affections et passions des hommes. Et nonobstant que ce ne soit ma profession de composer en ryme, ce neantmoins pour donner quelque grace à l'œuvre, et aussi en suyvant mon aucteur, j'ay observé les nombres de ses mettres autant qu'i m'a esté possible, et y ay adjousté rythme telle quelle. À laquelle il vous plaira n'avoir grand egard, mais seullement à la disposition de l'aucteur et merveilleuse œconomie. Car de moy je ne suys que son simple truchement fidele pour certain autant qu'i m'a esté possible, mais non suffisamment exercité en l'un et l'aultre langaige pour me debvoir paragonner à luy. Ce neantmoins, Sire, si je m'apperçoy que mon labeur vous soit agreable, j'auray pour le temps advenir plus grande promptitude à faire pour vous quelque meilleure chose. Aydant le Createur, lequel par sa saincte grace vous vueille donner tresbonne et longue vie.

\section{Argomento}

\section{ARGUMENT ET MATIERE DE LA PRESENTE TRAGEDIE}

Agamemnon estant au port de Aulide, allant à la chasse, print ung cerf ou une bische qui estoit à Diana, de quoy la deesse courroucee fist arrester les ventz, en sorte que l'armee des Grecz ne pouvoit passer la mer pour aller en Asie assieger Troye la grand. Par quoy les augures et divinateurs consultez du remede, respondirent qu'il failloit (pour appaiser Diana) qu'Agamemnon luy immolast sa fille Iphigenia. Ce qu'il fit. De quoy Clytemnestra sa femme courroucee, aussi pour la longue demeure de son absence, 
se enamoura de Egistus. Aprés que Troye fut destruicte, Agamemnon fut tué de sa femme et de Egistus à son retour, luy estant à soupper. Quoy voyant Electra fille de Agamemnon, ayant craincte que l'on ne tuast aussi son frere Orestés, le bailla à ung Pedagogue pour le mener hors le pays, et le saulver. Ce que fut faict. Vingt ans aprés, Orestés et son compaignon Pyladés, guidez du Pedagogue, retournerent en leur maison, qui estoit en Argie et Mycenes, et forgerent faulses nouvelles de la mort de Orestés et faignirent en apporter les ossemens en ung vaisseau. Où trouverent la mere Clytemnestra sans Egistus: lequel estoit allé aux champs passer le temps, et ilz la tuerent. Puys Egistus retourné, luy monstrerent sa femme morte et couverte. Et luy pensoit que ce fust le corps de Orestés. Mais à la descouvrir, bien congneut qu'il estoit surpris: et là par Orestés fut occis au propre lieu où il avoit tué son pere Agamemnon. Mais qui vouldra veoir une chose tres artificiellement faicte, veoye et contemple la recongnoissance de Orestés et d'icelle Electra, et devant les arguz et reprehensions de Electra, et de sa mere la royne Clytemnestra.

\section{Dedica al lettore}

\section{AU LECTEUR ${ }^{18}$}

LA chose est certeine, lecteur, Sans qu'aultre que moy te le die, A bien translater, qu'ung autheur Rendre doibt par son estudie En tant bon sens. La tragedie De Sophocle a sens fort suttil En grec, croy qu'ung esprit gentil Bien ne la tourna sans grand peine: A bon sens rendre, sçaiches qu'il Y Fault prudence bien certaine.

BIBLIOGRAFIA

\section{Bibliografia del corpus}

Le grandi edizioni cinquecentesche delle tragedie sofoclee, che precedono Lazare de Baïf sono: l'Aldina (1502); la Giuntina (1522).

[J. CAMERARIUS], Sophokleous Tragoidiai hepta. Sophoclis tragcedice septem cum commentarijs interpretationum argumenti Thebaidos fabularum Sophoclis, authore Ioachimo Camerario qu. iam recens natis atque ceditis, Hagenau, Johann Erben Setzer, 1534. 


\section{Manoscritto}

L'Electra di Baïf si trova in:

BAÏF, Lazare de, Tragedie de Sophoclés intitulee Electra, ms. Venezia, Biblioteca Marciana, XXIV

[=235] (CIV. 8: fondo Recanati IX).

\section{Edizioni antiche}

BÄ̈F, Lazare de, Tragedie de Sophoclés intitulee Electra [...], Paris, Estienne Roffet, 1537.

\section{Edizioni moderne}

BAÏF, Lazare de, Tragedie de Sophoclés intitulee Electra, a cura di F. FASSINA, Vercelli, Mercurio, 2012.

\section{Opere bio-bibliografiche concernenti Lazare de Baïf}

BOURILLY, Victor-Louis, Lazare de Baïf maître des Requêtes in AA. VV., Mélanges offerts à M. Emile Picot, Paris, Librairie Damascène Morgand, 1913, pp. 121-134.

BRUNEL, Pierre, Le mythe d'Electre, Paris, Champion, 1971.

BUDÉ, Guillaume, Opera omnia, Basileæ, apud Nicolaum Episcopium, 1557.

CHAMARD, Henri, Histoire de la Pléiade, Paris, Didier, 1939-1940.

CHAVY, Paul, Traducteurs d'autrefois: Moyen Age et Renaissance. Dictionnaire des traducteurs et de la littérature traduite en ancien et moyen français, Paris-Genève, Champion-Slatkine, 1988.

Dictionnaire des lettres françaises. Le XVIe siècle, sous la direction du cardinal G. GRENTE, édition revue et mise à jour sous la direction de M. SIMONIN, Paris, Fayard, («La Pochothèque»), 2001. [ERASMO], Opus epistolarum Desiderii Erasmi Roterodami, denuo recognitum et auctum per P. S. ALLEN, H. M. ALLEN et H. W. GERROD, Oxford, At the University Press, 1906-1965.

HAURÉAU, Barthélemy, Histoire littéraire du Maine, Paris, Dumoulin, 1870², t. I, pp. 227-245.

LA GARANDERIE, Marie-Madeleine de, L'approche philologique du fait antique in AA. VV., Actes du IXe Congrès de l'Association Guillaume Budé, Paris, Les Belles Lettres, 1975, pp. 705-714.

LA GARANDERIE, Marie-Madeleine de, voce «Lazare de BAïF» in Contemporaries of Erasmus. A biographical register of the Renaissance and Reformation, ed. P. G. BIETENHOLZ, Th. B. DEUTSCHER, Toronto-Buffalo-London, University of Toronto Press, 1985-1987, vol. 1, pp. 87-88.

PINVERT, Lucien, Lazare de Baïf, Paris, Albert Fontemoing, 1900.

Répertoire des Humanistes (XIVe-XVIIe siècles), répertoire établi par J.-F. MAILLARD, J. KECSKEMÉTI et M. PORTALIER, Paris-Turnhout, CNRS-Brepols, 1995, p. 49.

SAULNIER, Verdun-Louis, voce "BAÏF (Lazare $\mathrm{I}^{\mathrm{er}} \mathrm{de}$ )», in Dictionnaire de biographie française, sous la direction de J. BALTEAU, M. BARRoux, M. PRÉVOST; puis de M. PRÉVOST et R. D’AMAT, Paris, Letouzey, a partire dal 1933. 


\section{Bibliografia critica sul problema della traduzione}

AA. Vv., Traduction et adaptation en France à la fin du Moyen Âge et à la Renaissance, «Actes du Colloque de Nancy II (23-25 mars 1995)», réunis et présentés par C. BRUCKER, Paris, Champion, 1997.

ARIANI, Marco, Tra classicismo e manierismo. Il teatro tragico del Cinquecento, Firenze, Olschki, 1974. BALSAMO, Jean, Les traducteurs français d'ouvrages italiens et leurs mécènes (1574-1589), in AA. Vv., Le livre dans l'Europe de la Renaissance, "Actes du XXVIII ${ }^{e}$ Colloque International d'Études Humanistes de Tours», Nantes, Promodis-Éditions du Cercle de la librairie, 1988, pp. 122-132.

BIANCIOTTO, Gabriel, Langue conditionnée de traductions et modèles stylistiques au XVe siècle, in AA. Vv., Sémantique lexicale et sémantique grammaticale du moyen français, «Actes du Colloque de Bruxelles, Bruxelles (28-29 septembre 1978)», V.U.B., Centrum Voor Tall-en Literatuur- Wetenschat, 1978.

BRULARD, I., La traduction comme miroir d'un changement dans l'approche du sens à la Renaissance, «Cahiers de Linguistique de Louvain», 15, 1 (1990), pp. 13-21.

BRUNEAU, Charles, La phrase des traducteurs au XVI siècle, in AA. Vv., Mélanges d'histoire littéraire de la Renaissance offerts à Henri Chamard, Paris, Nizet, 1951, pp. 275-284.

CALVY DE LA FONTAINE, L'Antigone de Sophoclés, a cura di M. MASTROIANNI, Alessandria, Edizioni dell'Orso, 2000.

CATFORD, John Cunnison, A linguistic theory of translation, London, Oxford University Press, 1965.

CECCHETTI, Dario, Thomas Sébillet e la traduzione: $i$ testi proemiali dell' «Iphigéne d'Euripide», in Aa. Vv., Il progetto e la scrittura - Le projet de l'écriture, introduzione a cura di F. BRUERA, A. EMINA,

A. P. MOSSETTO, Roma, Bulzoni, 2007.

CHAvY, Paul, Les traductions humanistes au début de la Renaissance française: traductions médiévales, traductions modernes, «Canadian Review of Comparative Literature», VIlI-2 (1981), pp. 284-306.

DELCOURT, Marie, Étude sur les traductions des tragiques grecs et latins en France depuis la Renaissance, Bruxelles, M. Lamertin, 1925.

DOLET, Étienne, La maniere de bien traduire d'une langue en aultre d'advantage. De la punctuation de la langue françoyse. Plus des accents d'ycelle, Lyon, E. Dolet, 1540 (Genève, Slatkine Reprints) 1972.

DolET, Étienne, dans Préfaces françaises, par C. LONGEON, Genève, Droz, 1979.

Du Bellay, Joachim, La Deffence et Illustration de la Langue Francoyse, édition critique par H. CHAMARD, Paris, Didier, 1948.

Du BELlAY, Joachim, La Deffence, et illustration de la langue françoyse, édition et dossier critique par J.-C. MONFERRAN, Genève, Droz, 2001.

Du BELLAY, Joachim, Euvres complètes: I. La Deffence, et illustration de la langue françoyse, par F. GOYET et O. MILLET, Paris, Champion, 2003.

ESTIENNE, Henri, Project du livre intitulé de la precellence du langage françois, Paris, Mamert Patisson, 1579.

ESTIENNE, Henri, Conformité du langage françois avec le grec, éd. L. FEUGÈRE, Paris, J. Delalain, 1853, (Genève, Slatkine Reprints, 1970).

ESTIENNE, Robert, La maniere de tourner en Langue françoise les verbes actifz, passifz, gerundifz, supins \& participes, Paris, Robert Estienne, 1528. 
FoLENA, Gianfranco, Volgarizzare e tradurre, Torino, Einaudi, 1991.

FRITZ, Kurt von, Antike und Moderne Tragödie, Berlin, Neun Abhandlungen, 1962.

FUMAROLI, Marc, L'Âge de 1'éloquence: rhétorique et «res literaria» de la Renaissance au seuil de 1'époque classique, Genève, Droz, 1980.

GARNIER, Bruno, Pour une poétique de la traduction. L'«Hécube» d'Euripide en France de la traduction humaniste à la tragédie classique, Paris, L’Harmattan, 1999.

GUILLERM, Luce, L'auteur, les modèles et le pouvoir ou la topique de la traduction au XVIe siècle, «Revue des Sciences Humaines», 52 (1980), pp. 5-31.

GUILLERM, Luce, Sujet de l'écriture et traduction autour de 1540, Lille-Paris, Atelier national reproduction des thèses - Diffusion aux amateurs des livres, 1988.

HoRn-MONVAL, Madeleine, Répertoire bibliographique des traductions et adaptations françaises du théâtre étranger du XV siècle à nos jours, t. I, Théâtre grec antique, t. II, Théâtre latin, t. III, Théâtre italien, Paris, C.N.R.S., 1958-1960.

JouANNA, Arlette, La France du XVI siècle (1483-1598), Paris, P.U.F., 1996.

LA TAILlE, Jean de, De l'art de la tragédie (1572), éd. F. WEST, Manchester, At the University Press, 1939.

LAWTON, Harold Walter, Handbook of French Renaissance Dramatic Theory, Manchester, At the University Press, 1949.

LAZARD, Madeleine, Le théâtre en France au XVI siècle, Paris, P.U.F., 1980.

LEBÈGUE, Raymond, La langue des traducteurs français au XVI siècle, in AA. Vv., Festgabe Ernst Gamillscheg, Tübingen, Max Niemeyer Verlag, 1952, pp. 24-30.

LEBLANC, Paulette, Les écrits théoriques et critiques français des années 1540-1561 sur la tragédie, Paris, Nizet, 1972.

Mastroianni, Michele, Le «Antigoni» sofoclee del Cinquecento francese, Firenze, Olschki, 2004.

MASTROIANNI, Michele, Il genere tragico come luogo del sincretismo rinascimentale. L' «Antigone» de Robert Garnier, in AA. VV., Le syncrétisme pagano-chrétien à l'époque de l'Humanisme et de la Renaissance, «Actes du Colloque International de Chambéry, 16-17 mai 2002», publiés sous la direction de S. LARDON, «Franco-Italica», 25-26 (2004), pp. 199-232.

MASTROIANNI, Michele, $L$ '«interpretatio» dei cori nei primi volgarizzamenti francesi di tragedie greche, in A.A. V.V., Le scritture e le riscritture. Discorso religioso e discorso letterario in Europa nella prima età moderna, a cura di D. BORGOGNI e R. CAMERLENGO, Napoli, E.S.I., 2005, pp. 37-79.

MASTROIANNI, Michele, Lungo i sentieri del tragico. La rielaborazione teatrale in Francia dal Rinascimento al Barocco, Vercelli, Edizioni Mercurio, 2009

MASTROIANNI, Michele, Traduction des textes anciens et élaboration linguistique à la Renaissance française. Quelques aspects de la langue de l'«Antigone» de Calvy de La Fontaine, «Studi Francesi», 136 (2002).

Mathieu, Abel, Devis de la langue françoyse, Paris, Richard Breton, 1559.

MATHIEU, Abel, Second devis, et principal propos de la langue françoyse, Paris, Riehard Breton, 1560. 
MAZOUER, Charles, Les mythes antiques dans la tragédie française du XVI siècle, in AA. Vv., L'imaginaire du changement en France au XVI siècle, éd. C.-G. DUBoIs, Bordeaux, Presses Universitaires de Bordeaux, 1984, t. I, pp. 133-161.

MILLET, Olivier, De l'erreur au péché: la culpabilité dans la tragédie humaniste du XVI siècle, «Travaux de littérature», VIII (1995), pp. 57-73.

MounIN, Georges, La langue des traducteurs français au XVI siècle, in AA. Vv., Festgabe Ernst Gamillscheg, Tübingen, Max Niemeyer Verlag, 1952, pp. 24-30.

Mounin, Georges, Teoria e storia della traduzione, Torino, Einaudi, 1965.

NORTON, Glyn P., Translation Theory in Renaissance France: Etienne Dolet and the Rhetorical Tradition, «Renaissance and Reformation», 11 (1975), pp. 30-44.

NORTON, Glyn P., The Ideology and Language of Translation in Renaissance France and their Humanist Antecedents, Genève, Droz, 1984.

NORTON, Glyn P., Fidus interpres: a Philological Contribution to the Philosophy of Translation in Renaissance France, in AA. Vv., Neo-Latin and the Vernacular in Renaissance France, ed. T. C. CAVE and G. CASTOR, Oxford, At the Clarendon Press, 1984, pp. 252-272.

Peletier Du MANs, Jacques, L'art poetique françois (1555), éd. crit. d'après l'original avec introduction et notes de A. BOULANGER, Paris, Les Belles Lettres, 1930.

Ramus, Pierre, Ciceronianus, Paris, A. Wechel, 1557.

REVERDIN, Olivier, Les premiers cours de grec au Collège de France, Paris, P.U.F., 1984.

SÉBILLET, Thomas, Art poétique françois, éd. P. GAIFFE, Paris, Droz, 1932.

SonNINO, Lee A., A handbook to sixteenth-century rhetoric, London, Routledge and K. Paul, 1968.

STUREL, René, Essai sur les traductions du théâtre grec en français avant 1550, «Revue d'Histoire Littéraire de la France», 20 (1913), pp. 269-296 e 637-666.

VAuquelin De La Fresnaye, Jean, Art poétique, éd. G. Pellissier, Paris, Garnier, 1885 (Genève, Slatkine Reprints, 1970).

WEINBERG, Bernard, Critical Prefaces of the French Renaissance, Evanston (Ill.), Northwestern University Press, 1950.

WORTH, Valerie, Practising Translation in Renaissance France, Oxford, At the Clarendon Press, 1988. ZUBER, Roger, «Les Belles Infidèles» et la formation du goût classique, Paris, Colin, 1968.

\section{NOTE}

1. Sulla biografia di Lazare de Baiff sono ancor oggi fondamentali: L. Pinvert, Lazare de Baïf, Paris, Albert Fontemoing, 1900; V.-L. Saulnier, voce «baïf (Lazare $\mathrm{I}^{\mathrm{er}}$ de)», in Dictionnaire de biographie française, sous la direction de J. Balteau, M. Barroux, M. Prévost; puis de M. Prévost et R. d'Amat, Paris, Letouzey, a partire dal 1933; H. Chamard, Histoire de la Pléiade, Paris, Didier, 1939-1940 (qui vol. I, passim); M.-M. de La Garanderie, voce «Lazare de BAÏF» in Contemporaries of Erasmus. A biographical register of the Renaissance and Reformation, ed. P.G. Bietenholz, T.B. Deutscher, Toronto-Buffalo-London, University of Toronto Press, 1985-1987, vol. 1, pp. 87-88. Per la corretta grafia del nome Baïf e per le origini della famiglia, cfr. L. Saulnier, art. cit. 
2. Ms. BN fr. 1396 (conviene forse ricordare che non tutti gli studiosi sono d'accordo sulla paternità di quest'opera).

3. Ms. BN fr. 3941, f. 215 r.

4. È del tutto smentita l'attribuzione a Lazare della traduzione dell'Ecuba di Euripide (1544), sicuramente opera di Bochetel. A questo proposito, cfr. R. Sturel, A propos d'un manuscrit du Musée Condé, in Mélanges offerts à M. Emile Châtelain, Paris, Champion, 1910; M. Delcourt, Étude sur les traductions des tragiques grecs et latins en France depuis la Renaissance, Bruxelles, M. Lamertin, 1925, pp. 26-33; в. Garnier, Pour une poétique de la traduction. L'«Hécube» d'Euripide en France de la traduction humaniste à la tragédie classique, Paris, L'Harmattan, 1999, pp. 28-30.

5. Cfr. Chronologie in Ronsard, Euvres complètes, édition établie, présentée et annotée par J. Céard,

D. Ménager et M. Simonin, Paris, Gallimard, «La Pléiade», 1993-1994, vol. I, pp. XLVII.

6. Cfr. Ibid., p. XLVIII.

7. Euripidis Hecuba et Iphigeneia latince factoe Erasmo interprete, ed. J. H. Waszink, in Desiderii Erasmi Roterodami, Opera omnia, I-1, Amsterdam, North-Holland Publishing Company, 1969, pp. 193-359 (ed. orig.: Paris, J. Badius, 1506).

8. [J. Camerarius], Sophokleous Tragoidiai hepta. Sophoclis trageodice septem cum commentarijs interpretationum argumenti Thebaidos fabularum Sophoclis, authore Ioachimo Camerario qu. iam recens natis atque ceditis, Hagenau, Johann Erben Setzer, 1534. Joachim Liebhard (1500-1574), indicato comunemente in Germania con l'appellativo di Kammermeister, è generalmente noto in Europa come Camerarius.

9. Ep. 188 (ed. Allen), in Desiderii Erasmi Roterodami, Opera omnia, I-1, cit., p. 218.

10. Cfr. Euripidis Hecuba et Iphigeneia latince factee Erasmo interprete, cit., p. 217 (lettera dedicatoria dell'Hecuba a William Warham): «Adde nunc choros nescio quanam affectione adeo obscuros, ut Edipo quopiam aut Delio sit opus magis quam interprete».

11. Cfr. i lavori di Michele Mastroianni e di Bruno Garnier sull'interpretatio della tragedia antica nel Cinquecento francese: in particolare M. Mastroianni, Le "Antigoni» sofoclee del Cinquecento francese, Firenze, Olschki, 2004; id., Il genere tragico come luogo del sincretismo rinascimentale. L'«Antigone» di Robert Garnier, in id., Lungo i sentieri del tragico. La rielaborazione teatrale in Francia dal Rinascimento al Barocco, Vercelli, Edizioni Mercurio, 2009, pp. 59-101; id., L'«interpretatio» dei cori nei primi volgarizzamenti francesi di tragedie greche, ibid., pp. 13-57; B.Garnier, op. cit. Cfr. anche C. Mazouer, Le Théâtre français de la Renaissance, Paris, Champion, 2002, pp. 196-211.

12. Cfr. D. Cecchetti, Thomas Sébillet e la traduzione: $i$ testi proemiali dell'«Iphigéne d'Euripide», in AA. VV., Il progetto e la scrittura - Le projet de l'écriture, introduzione a cura di F. Bruera, A. Emina, A. P. Mossetto, Roma, Bulzoni, 2007, pp. 29-55.

13. Per un approfondimento sulla metrica di Lazare de Baïf analizzata in parallelo con l'originale sofocleo, cfr. l'edizione di riferimento: Lazare de Baïf, Tragédie de Sophoclés intitulee Electra, a cura di F. Fassina, Vercelli, Mercurio, 2011.

14. Cfr. D. Cecchetti, Thomas Sébillet e la traduzione ecc., cit., § 3 .

15. Il prologo compare soltanto nel manoscritto: anche per la trascrizione di questa breve sezione abbiamo adottato i criteri sopra enunciati. Nella trascrizione dell'Argument adottiamo la grafia Agamemnon in luogo di Agamenon, ritenendo che il tipografo abbia, per svista, omesso la tilde.

16. La Diffinition compare in ms. al termine della tragedia (44v) con il titolo Que c'est que Tragedie.

17. Il Prologue è omesso nell'ed.

18. L'indirizzo Au lecteur non compare nel ms. 


\section{RIASSUNTI}

I paratesti, che qui analizziamo, comprendono: titoli, privilegio, un breve discorso definitorio concernente il genere tragico, un prologo che illustra le finalità e le modalità del volgarizzamento, l'argomento della tragedia, una dedica al lettore in dieci ottosillabi a rima alternata.

\section{INDICE}

Indice geografico : domaine français

Mots-clés : translation, traduction, traduction ligne pour ligne, traduction vers pour vers, nombre, mètre, rythme, tragédie, moralité

indice cronologico XVIe siècle

\section{AUTORI}

\section{FILIPPO FASSINA}

Filippo Fassina, dottore di ricerca in Francesistica, è assegnista (boursier) nel Dipartimento di Studi Umanistici dell'Università del Piemonte Orientale. Si occupa di volgarizzamenti e riscritture francesi di tragedie classiche fra Cinque e Settecento. 